\title{
THE COMPARATIVE STUDY OF SPOKEN-LANGUAGE PROCESSING
}

\author{
Anne Cutler \\ Max-Planck-Institute for Psycholinguistics \\ Wundtlaan 1 \\ 6525 XD Nijmegen \\ Netherlands
}

\begin{abstract}
Psycholinguists are saddled with a paradox. Their aim is to construct a model of human language processing, which will hold equally well for the processing of any language, but this aim cannot be achieved just by doing experiments in any language. They have to compare processing of many languages, and actively search for effects which are specific to a single language, even though a model which is itself specific to a single language is really the last thing they want.
\end{abstract}

\section{SUMMARY}

Spoken-language processing, from the psycholinguist's point of view, means the operations which occur in speaking, between conceiving the intention to communicate a message and producing an utterance, and in listening, between a speech signal impinging upon the ear and the reconstruction of a message. But speaking and listening take place in a particular language. The characteristics of the language at all structural levels inevitably play a role in the processing - so the model has to take account of how processing may be affected by whether a language has tone, fixed stress, pitch accent, vowel harmony, determiners, copula, prefixes, suffixes, infixes, noun classifiers, SVO word order, OSV word order, or any other of a very long list of properties.

This all makes life extremely interesting for the psycholinguist but in the long run it is not what must be modelled. Psycholinguists might be happy producing explicit models of how to undertand and speak English, Mandarin, Sesotho and so on, if these indeed appeared to be fundamentally different achievements of the human mind; but it is clear that that they are not. A child is born with the ability to acquire language, but not any particular language: just whatever language is spoken in the environment. The characteristics of the language may affect the acquisition process even as they affect production and perception, so that different aspects of linguistic structure may for instance be acquired in differing order in one language compared with another. But whatever the language, children master its essentials fully within a few years, and we know of no evidence that human languages differ in how intrinsically difficult they are to process in any sense speak, understand, acquire. Thus an adequate model of spokenlanguage processing should concentrate on the universal features which hold true of processing in every language.

Alas, this does not mean that confining psycholinguistic experiments to the universal features which hold true of the structure of every language will produce the desired universal model of processing. But it likewise does not mean that experiments on language-specific structure should be avoided because they may be irrelevant to the universal model.

These points can be clearly illustrated by considering, as this plenary lecture will do, psycholinguistic studies of the recognition of continuous speech by human listeners. In no known language do speakers introduce a pause between the lexical units of which utterances are composed (unlike orthographies, of course, which very often do signal word boundaries explicitly). Speech recognition must therefore involve, for every language, the necessity of identifying on-line where words begin and end, i.e. segmenting utterances into their component parts.

Suppose we address the segmentation issue by seeking a structural level with relevance to the phonological description of any language - for example, the syllable. An experiment on the role of the syllable in the segmentation of continuous speech is not difficult to design, and indeed psycholinguists have carried out many such studies. The results clearly demonstrate that it is not possible to determine the universal role of such a universal construct by experiments in any randomly chosen language because the syllable has been shown to play a differing role across languages. The same is true, as this lecture will describe, of many other phonological commonalities between languages, from coarticulatory information about segments to prosodic cues to syntax.

On the other hand, suppose we consider a structural feature which occurs in the phonology of relatively few languages - for example, vowel harmony. In Finnish, as recent studies by our research group have demonstrated, this feature provides information which native listeners exploit in segmenting speech. This is language-specific processing; but consideration of, for example, where Finnish listeners make use of vowel harmony information and where they ignore it, or why some kinds of vowel harmony information turn out to be more effective than others, can usefully constrain a language-universal model. Again, further available results show the same to be true of many other language-specific aspects of phonological structure, from vowel epenthesis through lexical stress to tone sandhi.

The segmentation of continuous speech by human listeners involves, on current evidence, a number of characteristics which may be captured with a general (i.e. universal) description which awaits for each language a language-specific implementation; the task of the psycholinguist is thus to find the universal thread which binds the language-particular data. 